\title{
Mathematical functions of the rate of enzymatic reaction with temperature, concentration of substrate and concentration of enzyme are proved that the divide differences are symmetrical in all their arguments
}

\author{
Nizam Uddin \\ M. B. Khalsa College, Indore-452002, Madhya Pradesh, India \\ E-mail: nizamuddin4research@gmail.com
}

\begin{abstract}
In this research paper, I used mathematical functions of the rate of enzymatic reaction for proving the divide differences are symmetrical in all their argument. The concentration of substrate is the limiting factor, as the substrate concentration increases, the Enzyme reaction rate increases. Assuming a sufficient concentration of substrate is available, increasing Enzyme concentration will increase the enzymatic reaction rate. The rise in Temperature accelerates an Enzyme reaction but at the same time causes inactivation of the protein. At certain Temperature known as the optimum Temperature the activity is maximum. Temperature, concentration of substrate and concentration of enzyme are increased the rate of enzymatic reaction at a limit which is called optimum limit. I take their values in mathematical form where " $n$ " is optimum limit.
\end{abstract}

Keywords: Concentration of enzyme, Concentration of substrate, Divide differences, Mathematical functions, rate of enzymatic reaction, Temperature

\section{Introduction}

Mathematical functions of the rate of enzymatic reaction with temperature, concentration of substrate and concentration of enzyme are $\boldsymbol{V}=\boldsymbol{f}(\boldsymbol{E}), \boldsymbol{V}=\boldsymbol{f}(\boldsymbol{S})$ and $\boldsymbol{V}=\boldsymbol{f}(\boldsymbol{T})$ [1]. These mathematical functions are show that "the concentration of substrate is the limiting factor, as the substrate concentration increases, the Enzyme reaction rate increases. Assuming a sufficient concentration of substrate is available, increasing Enzyme concentration will increase the rate of enzymatic reaction. Temperature, concentration of substrate and concentration of enzyme are increased the rate of enzymatic reaction at a limit which is called optimum limit [2]-[5]." Divide differences are symmetrical in all their arguments [6].

\section{Divide differences are symmetrical with $V=f(T)$}

If $\left(\boldsymbol{T}_{0}, \boldsymbol{V}_{0}\right),\left(\boldsymbol{T}_{1}, V_{1}\right) \ldots \ldots \ldots\left(\boldsymbol{T}_{n}, \boldsymbol{V}_{n}\right)$ denoted the values of the function where $\boldsymbol{V}$ is the rate of enzymatic reaction with Temperature then $\frac{\boldsymbol{V}_{1}-\boldsymbol{V}_{0}}{\boldsymbol{T}_{1}-\boldsymbol{T}_{0}} \frac{\boldsymbol{V}_{2}-\boldsymbol{V}_{1}}{\boldsymbol{T}_{2}-\boldsymbol{T}_{1}}, \frac{\boldsymbol{V}_{3}-\boldsymbol{V}_{2}}{\boldsymbol{T}_{3}-\boldsymbol{T}_{2}}, \frac{\boldsymbol{V}_{4}-\boldsymbol{V}_{3}}{\boldsymbol{T}_{4}-\boldsymbol{T}_{3}}, \ldots, \frac{\boldsymbol{V}_{n}-\boldsymbol{V}_{n-1}}{\boldsymbol{T}_{\boldsymbol{n}}-\boldsymbol{T}_{\boldsymbol{n}-1}}$ are called the divide differences of $\boldsymbol{T}$. We know that the first divide difference with two arguments $\boldsymbol{T}_{0}, \boldsymbol{T}_{1}$ is given by

$\boldsymbol{f}\left(\boldsymbol{T}_{0}, \boldsymbol{T}_{1}\right)=\frac{\boldsymbol{f}\left(\boldsymbol{T}_{1}\right)-\boldsymbol{f}\left(\boldsymbol{T}_{0}\right)}{\boldsymbol{T}_{1}-\boldsymbol{T}_{0}}$

$\boldsymbol{f}\left(\boldsymbol{T}_{0}, \boldsymbol{T}_{1}\right)=\frac{\boldsymbol{f}\left(\boldsymbol{T}_{0}\right)}{\boldsymbol{T}_{0}-\boldsymbol{T}_{1}}+\frac{\boldsymbol{f}\left(\boldsymbol{T}_{1}\right)}{\boldsymbol{T}_{1}-\boldsymbol{T}_{0}}$

Again second difference with three arguments $\boldsymbol{T}_{0}, \boldsymbol{T}_{1}, \boldsymbol{T}_{2}$ is given by

$\boldsymbol{f}\left(\boldsymbol{T}_{0}, \boldsymbol{T}_{1}, \boldsymbol{T}_{2}\right)=\frac{\boldsymbol{f}\left(\boldsymbol{T}_{1}, \boldsymbol{T}_{2}\right)-\boldsymbol{f}\left(\boldsymbol{T}_{0}, \boldsymbol{T}_{1}\right)}{\boldsymbol{T}_{2}-\boldsymbol{T}_{0}}$ 


$$
\begin{aligned}
\boldsymbol{f}\left(\boldsymbol{T}_{0}, \boldsymbol{T}_{1}, \boldsymbol{T}_{2}\right) & =\frac{\boldsymbol{f}\left(\boldsymbol{T}_{0}, \boldsymbol{T}_{1}\right)}{\boldsymbol{T}_{0}-\boldsymbol{T}_{2}}+\frac{\boldsymbol{f}\left(\boldsymbol{T}_{1}, \boldsymbol{T}_{2}\right)}{\boldsymbol{T}_{2}-\boldsymbol{T}_{0}} \\
\boldsymbol{f}\left(\boldsymbol{T}_{0}, \boldsymbol{T}_{1}, \boldsymbol{T}_{2}\right) & =\left[\boldsymbol{f}\left(\boldsymbol{T}_{0}, \boldsymbol{T}_{1}\right)-\boldsymbol{f}\left(\boldsymbol{T}_{1}, \boldsymbol{T}_{2}\right)\right] \frac{1}{\boldsymbol{T}_{0}-\boldsymbol{T}_{2}} \\
\boldsymbol{f}\left(\boldsymbol{T}_{0}, \boldsymbol{T}_{1}, \boldsymbol{T}_{2}\right) & =\left[\frac{\boldsymbol{f}\left(\boldsymbol{T}_{1}\right)-\boldsymbol{f}\left(\boldsymbol{T}_{0}\right)}{\boldsymbol{T}_{1}-\boldsymbol{T}_{0}}-\frac{f\left(\boldsymbol{T}_{2}\right)-\boldsymbol{f}\left(\boldsymbol{T}_{1}\right)}{\boldsymbol{T}_{2}-\boldsymbol{T}_{1}}\right] \frac{1}{\boldsymbol{T}_{0}-\boldsymbol{T}_{2}} \\
\boldsymbol{f}\left(\boldsymbol{T}_{0}, \boldsymbol{T}_{1}, \boldsymbol{T}_{2}\right) & =\left[\frac{\boldsymbol{f}\left(\boldsymbol{T}_{0}\right)}{\boldsymbol{T}_{1}-\boldsymbol{T}_{0}}-\frac{f\left(\boldsymbol{T}_{0}\right)}{\boldsymbol{T}_{1}-\boldsymbol{T}_{0}}+\frac{\boldsymbol{f}\left(\boldsymbol{T}_{1}\right)}{\boldsymbol{T}_{2}-\boldsymbol{T}_{1}}-\frac{\boldsymbol{f}\left(\boldsymbol{T}_{2}\right)}{\boldsymbol{T}_{2}-\boldsymbol{T}_{1}}\right] \frac{1}{\boldsymbol{T}_{0}-\boldsymbol{T}_{2}} \\
\boldsymbol{f}\left(\boldsymbol{T}_{0}, \boldsymbol{T}_{1}, \boldsymbol{T}_{2}\right) & =\frac{\boldsymbol{f}\left(\boldsymbol{T}_{0}\right)}{\left(\boldsymbol{T}_{0}-\boldsymbol{T}_{1}\right)\left(\boldsymbol{T}_{0}-\boldsymbol{T}_{2}\right)}-\frac{\boldsymbol{f}\left(\boldsymbol{T}_{1}\right)}{\left(\boldsymbol{T}_{1}-\boldsymbol{T}_{0}\right)\left(\boldsymbol{T}_{1}-\boldsymbol{T}_{2}\right)}+\frac{\boldsymbol{f}\left(\boldsymbol{T}_{1}\right)}{\left(\boldsymbol{T}_{1}-\boldsymbol{T}_{0}\right)\left(\boldsymbol{T}_{2}-\boldsymbol{T}_{1}\right)}
\end{aligned}
$$

Similarly

$$
\begin{aligned}
\boldsymbol{f}\left(\boldsymbol{T}_{0}, \boldsymbol{T}_{1}, \boldsymbol{T}_{2}, \boldsymbol{T}_{3}\right)= & \frac{\boldsymbol{f}\left(\boldsymbol{T}_{0}\right)}{\left(\boldsymbol{T}_{0}-\boldsymbol{T}_{1}\right)\left(\boldsymbol{T}_{0}-\boldsymbol{T}_{2}\right)\left(\boldsymbol{T}_{0}-\boldsymbol{T}_{2}\right)}+\frac{f\left(\boldsymbol{T}_{1}\right)}{\left(\boldsymbol{T}_{1}-\boldsymbol{T}_{0}\right)\left(\boldsymbol{T}_{1}-\boldsymbol{T}_{2}\right)\left(\boldsymbol{T}_{1}-\boldsymbol{T}_{2}\right)}+\frac{f\left(\boldsymbol{T}_{2}\right)}{\left(\boldsymbol{T}_{2}-\boldsymbol{T}_{0}\right)\left(\boldsymbol{T}_{2}-\boldsymbol{T}_{1}\right)\left(\boldsymbol{T}_{2}-\boldsymbol{T}_{3}\right)} \\
& +\frac{f\left(\boldsymbol{T}_{3}\right)}{\left(\boldsymbol{T}_{3}-\boldsymbol{T}_{0}\right)\left(\boldsymbol{T}_{3}-\boldsymbol{T}_{1}\right)\left(\boldsymbol{T}_{3}-\boldsymbol{T}_{2}\right)}
\end{aligned}
$$

And in general

$$
f\left(\boldsymbol{T}_{0}, \boldsymbol{T}_{1}, \boldsymbol{T}_{2}, \ldots, \boldsymbol{T}_{n}\right)=\frac{f\left(\boldsymbol{T}_{0}\right)}{\left(\boldsymbol{T}_{0}-\boldsymbol{T}_{1}\right)\left(\boldsymbol{T}_{0}-\boldsymbol{T}_{2}\right) \ldots\left(\boldsymbol{T}_{0}-\boldsymbol{T}_{n}\right)}+\frac{\boldsymbol{f}\left(\boldsymbol{T}_{1}\right)}{\left(\boldsymbol{T}_{1}-\boldsymbol{T}_{0}\right)\left(\boldsymbol{T}_{1}-\boldsymbol{T}_{2}\right) \ldots\left(\boldsymbol{T}_{1}-\boldsymbol{T}_{n}\right)}+\ldots+\frac{f\left(\boldsymbol{T}_{n}\right)}{\left(\boldsymbol{T}_{n}-\boldsymbol{T}_{0}\right)\left(\boldsymbol{T}_{n}-\boldsymbol{T}_{1}\right) \ldots\left(\boldsymbol{T}_{n}-\boldsymbol{T}_{n-1}\right)}
$$

\section{Divide differences are symmetrical with $V=f(S)$}

If $\left(\boldsymbol{S}_{0}, \boldsymbol{V}_{0}\right),\left(\boldsymbol{S}_{1}, \boldsymbol{V}_{1}\right) \ldots\left(\boldsymbol{S}_{n}, \boldsymbol{V}_{n}\right)$ denoted the values of the function where $\boldsymbol{V}$ is the rate of enzymatic reaction with concentration of substrate then $\frac{V_{1}-V_{0}}{S_{1}-S_{0}} \frac{V_{2}-V_{1}}{S_{2}-S_{1}}, \frac{V_{3}-V_{2}}{S_{3}-S_{2}}, \frac{V_{4}-V_{3}}{S_{4}-S_{3}}, \ldots, \frac{V_{n}-V_{n-1}}{S_{n}-S_{n-1}}$ are called the divide differences of

$S$. We know that the first divide difference with two arguments $S_{0}, S_{1}$ is given by

$$
\begin{aligned}
& \boldsymbol{f}\left(\boldsymbol{S}_{0}, \boldsymbol{S}_{1}\right)=\frac{\boldsymbol{f}\left(\boldsymbol{S}_{1}\right)-\boldsymbol{f}\left(\boldsymbol{S}_{0}\right)}{\boldsymbol{S}_{1}-\boldsymbol{S}_{0}} \\
& \boldsymbol{f}\left(\boldsymbol{S}_{0}, \boldsymbol{S}_{1}\right)=\frac{\boldsymbol{f}\left(\boldsymbol{S}_{0}\right)}{\boldsymbol{S}_{0}-\boldsymbol{S}_{1}}+\frac{\boldsymbol{f}\left(\boldsymbol{S}_{1}\right)}{\boldsymbol{S}_{1}-\boldsymbol{S}_{0}}
\end{aligned}
$$

Again second difference with three arguments $\boldsymbol{S}_{0}, \boldsymbol{S}_{1}, \boldsymbol{S}_{2}$ is given by

$$
\begin{aligned}
& f\left(S_{0}, S_{1}, S_{2}\right)=\frac{f\left(S_{1}, S_{2}\right)-f\left(S_{0}, S_{1}\right)}{S_{2}-S_{0}} \\
& f\left(S_{0}, S_{1}, S_{2}\right)=\frac{f\left(S_{0}, S_{1}\right)}{S_{0}-S_{2}}+\frac{f\left(S_{1}, S_{2}\right)}{S_{2}-S_{0}} \\
& f\left(S_{0}, S_{1}, S_{2}\right)=\left[f\left(S_{0}, S_{1}\right)-f\left(S_{1}, S_{2}\right)\right] \frac{1}{S_{0}-S_{2}} \\
& f\left(S_{0}, S_{1}, S_{2}\right)=\left[\frac{f\left(S_{1}\right)-f\left(S_{0}\right)}{S_{1}-S_{0}}-\frac{f\left(S_{2}\right)-f\left(S_{1}\right)}{S_{2}-S_{1}}\right] \frac{1}{S_{0}-S_{2}} \\
& f\left(S_{0}, S_{1}, S_{2}\right)=\left[\frac{f\left(S_{0}\right)}{S_{1}-S_{0}}-\frac{f\left(S_{0}\right)}{S_{1}-S_{0}}+\frac{f\left(S_{1}\right)}{S_{2}-S_{1}}-\frac{f\left(S_{2}\right)}{S_{2}-S_{1}}\right] \frac{1}{S_{0}-S_{2}} \\
& f\left(S_{0}, S_{1}, S_{2}\right)=\frac{f\left(S_{0}\right)}{\left(S_{0}-S_{1}\right)\left(S_{0}-S_{2}\right)}-\frac{f\left(S_{1}\right)}{\left(S_{1}-S_{0}\right)\left(S_{1}-S_{2}\right)}+\frac{f\left(S_{1}\right)}{\left(S_{1}-S_{0}\right)\left(S_{2}-S_{1}\right)}
\end{aligned}
$$

Similarly 


$$
\begin{aligned}
\boldsymbol{f}\left(\boldsymbol{S}_{0}, \boldsymbol{S}_{1}, \boldsymbol{S}_{2}, \boldsymbol{S}_{3}\right) & =\frac{\boldsymbol{f}\left(\boldsymbol{S}_{0}\right)}{\left(\boldsymbol{S}_{0}-\boldsymbol{S}_{1}\right)\left(\boldsymbol{S}_{0}-\boldsymbol{S}_{2}\right)\left(\boldsymbol{S}_{0}-\boldsymbol{S}_{2}\right)}+\frac{\boldsymbol{f}\left(\boldsymbol{S}_{1}\right)}{\left(\boldsymbol{S}_{1}-\boldsymbol{S}_{0}\right)\left(\boldsymbol{S}_{1}-\boldsymbol{S}_{2}\right)\left(\boldsymbol{S}_{1}-\boldsymbol{S}_{2}\right)}+\frac{\boldsymbol{f}\left(\boldsymbol{S}_{2}\right)}{\left(\boldsymbol{S}_{2}-\boldsymbol{S}_{0}\right)\left(\boldsymbol{S}_{2}-\boldsymbol{S}_{1}\right)\left(\boldsymbol{S}_{2}-\boldsymbol{S}_{3}\right)} \\
& +\frac{\boldsymbol{f}\left(\boldsymbol{S}_{3}\right)}{\left(\boldsymbol{S}_{3}-\boldsymbol{S}_{0}\right)\left(\boldsymbol{S}_{3}-\boldsymbol{S}_{1}\right)\left(\boldsymbol{S}_{3}-\boldsymbol{S}_{2}\right)}
\end{aligned}
$$

And in general

$$
\boldsymbol{f}\left(\boldsymbol{S}_{0}, \boldsymbol{S}_{1}, \ldots, \boldsymbol{S}_{n}\right)=\frac{\boldsymbol{f}\left(\boldsymbol{S}_{0}\right)}{\left(\boldsymbol{S}_{0}-\boldsymbol{S}_{1}\right)\left(\boldsymbol{S}_{0}-\boldsymbol{S}_{2}\right) . .\left(\boldsymbol{S}_{0}-\boldsymbol{S}_{n}\right)}+\frac{\boldsymbol{f}\left(\boldsymbol{S}_{1}\right)}{\left(\boldsymbol{S}_{1}-\boldsymbol{S}_{0}\right)\left(\boldsymbol{S}_{1}-\boldsymbol{S}_{2}\right) . .\left(\boldsymbol{S}_{1}-\boldsymbol{S}_{n}\right)}+\ldots+\frac{f\left(\boldsymbol{S}_{n}\right)}{\left(\boldsymbol{S}_{n}-\boldsymbol{S}_{0}\right)\left(\boldsymbol{S}_{n}-\boldsymbol{S}_{1}\right) . .\left(\boldsymbol{S}_{n}-\boldsymbol{S}_{n-1}\right)}
$$

\section{Divide differences are symmetrical with $V=f(E)$}

If $\left(\boldsymbol{E}_{0}, \boldsymbol{V}_{0}\right),\left(\boldsymbol{E}_{1}, \boldsymbol{V}_{1}\right) \ldots\left(\boldsymbol{E}_{n}, \boldsymbol{V}_{n}\right)$ denoted the values of the function where $\boldsymbol{V}$ is the rate of enzymatic reaction with concentration of enzyme then $\frac{\boldsymbol{V}_{1}-\boldsymbol{V}_{0}}{\boldsymbol{E}_{1}-\boldsymbol{E}_{0}} \frac{\boldsymbol{V}_{2}-\boldsymbol{V}_{1}}{\boldsymbol{E}_{2}-\boldsymbol{E}_{1}}, \frac{\boldsymbol{V}_{3}-\boldsymbol{V}_{2}}{\boldsymbol{E}_{3}-\boldsymbol{E}_{2}}, \frac{\boldsymbol{V}_{4}-\boldsymbol{V}_{3}}{\boldsymbol{E}_{4}-\boldsymbol{E}_{3}}, \ldots, \frac{\boldsymbol{V}_{n}-\boldsymbol{V}_{n-1}}{\boldsymbol{E}_{n}-\boldsymbol{E}_{n-1}}$ are called the divide differences of $\boldsymbol{E}$. We know that the first divide difference with two arguments $\boldsymbol{E}_{0}, \boldsymbol{E}_{1}$ is given by

$\boldsymbol{f}\left(\boldsymbol{E}_{0}, \boldsymbol{E}_{1}\right)=\frac{\boldsymbol{f}\left(\boldsymbol{E}_{1}\right)-\boldsymbol{f}\left(\boldsymbol{E}_{0}\right)}{\boldsymbol{E}_{1}-\boldsymbol{E}_{0}}$

$\boldsymbol{f}\left(\boldsymbol{E}_{0}, \boldsymbol{E}_{1}\right)=\frac{\boldsymbol{f}\left(\boldsymbol{E}_{0}\right)}{\boldsymbol{E}_{0}-\boldsymbol{E}_{1}}+\frac{\boldsymbol{f}\left(\boldsymbol{E}_{1}\right)}{\boldsymbol{E}_{1}-\boldsymbol{E}_{0}}$

Again second difference with three arguments $\boldsymbol{E}_{0}, \boldsymbol{E}_{1}, \boldsymbol{E}_{2}$ is given by

$\boldsymbol{f}\left(\boldsymbol{E}_{0}, \boldsymbol{E}_{1}, \boldsymbol{E}_{2}\right)=\frac{\boldsymbol{f}\left(\boldsymbol{E}_{1}, \boldsymbol{E}_{2}\right)-\boldsymbol{f}\left(\boldsymbol{E}_{0}, \boldsymbol{E}_{1}\right)}{\boldsymbol{E}_{2}-\boldsymbol{E}_{0}}$

$\boldsymbol{f}\left(\boldsymbol{E}_{0}, \boldsymbol{E}_{1}, \boldsymbol{E}_{2}\right)=\frac{\boldsymbol{f}\left(\boldsymbol{E}_{0}, \boldsymbol{E}_{1}\right)}{\boldsymbol{E}_{0}-\boldsymbol{E}_{2}}+\frac{\boldsymbol{f}\left(\boldsymbol{E}_{1}, \boldsymbol{E}_{2}\right)}{\boldsymbol{E}_{2}-\boldsymbol{E}_{0}}$

$\boldsymbol{f}\left(\boldsymbol{E}_{0}, \boldsymbol{E}_{1}, \boldsymbol{E}_{2}\right)=\left[\boldsymbol{f}\left(\boldsymbol{E}_{0}, \boldsymbol{E}_{1}\right)-\boldsymbol{f}\left(\boldsymbol{E}_{1}, \boldsymbol{E}_{2}\right)\right] \frac{1}{\boldsymbol{E}_{0}-\boldsymbol{E}_{2}}$

$\boldsymbol{f}\left(\boldsymbol{E}_{0}, \boldsymbol{E}_{1}, \boldsymbol{E}_{2}\right)=\left[\frac{\boldsymbol{f}\left(\boldsymbol{E}_{1}\right)-\boldsymbol{f}\left(\boldsymbol{E}_{0}\right)}{\boldsymbol{E}_{1}-\boldsymbol{E}_{0}}-\frac{\boldsymbol{f}\left(\boldsymbol{E}_{2}\right)-\boldsymbol{f}\left(\boldsymbol{E}_{1}\right)}{\boldsymbol{E}_{2}-\boldsymbol{E}_{1}}\right] \frac{1}{\boldsymbol{E}_{0}-\boldsymbol{E}_{2}}$

$\boldsymbol{f}\left(\boldsymbol{E}_{0}, \boldsymbol{E}_{1}, \boldsymbol{E}_{2}\right)=\left[\frac{\boldsymbol{f}\left(\boldsymbol{E}_{0}\right)}{\boldsymbol{E}_{1}-\boldsymbol{E}_{0}}-\frac{\boldsymbol{f}\left(\boldsymbol{E}_{0}\right)}{\boldsymbol{E}_{1}-\boldsymbol{E}_{0}}+\frac{\boldsymbol{f}\left(\boldsymbol{E}_{1}\right)}{\boldsymbol{E}_{2}-\boldsymbol{E}_{1}}-\frac{\boldsymbol{f}\left(\boldsymbol{E}_{2}\right)}{\boldsymbol{E}_{2}-\boldsymbol{E}_{1}}\right] \frac{1}{\boldsymbol{E}_{0}-\boldsymbol{E}_{2}}$

$\boldsymbol{f}\left(\boldsymbol{E}_{0}, \boldsymbol{E}_{1}, \boldsymbol{E}_{2}\right)=\frac{\boldsymbol{f}\left(\boldsymbol{E}_{0}\right)}{\left(\boldsymbol{E}_{0}-\boldsymbol{E}_{1}\right)\left(\boldsymbol{E}_{0}-\boldsymbol{E}_{2}\right)}-\frac{\boldsymbol{f}\left(\boldsymbol{E}_{1}\right)}{\left(\boldsymbol{E}_{1}-\boldsymbol{E}_{0}\right)\left(\boldsymbol{E}_{1}-\boldsymbol{E}_{2}\right)}+\frac{\boldsymbol{f}\left(\boldsymbol{E}_{1}\right)}{\left(\boldsymbol{E}_{1}-\boldsymbol{E}_{0}\right)\left(\boldsymbol{E}_{2}-\boldsymbol{E}_{1}\right)}$

Similarly

$$
\begin{aligned}
\boldsymbol{f}\left(\boldsymbol{E}_{0}, \boldsymbol{E}_{1}, \boldsymbol{E}_{2}, \boldsymbol{E}_{3}\right) & =\frac{\boldsymbol{f}\left(\boldsymbol{E}_{0}\right)}{\left(\boldsymbol{E}_{0}-\boldsymbol{E}_{1}\right)\left(\boldsymbol{E}_{0}-\boldsymbol{E}_{2}\right)\left(\boldsymbol{E}_{0}-\boldsymbol{E}_{2}\right)}+\frac{\boldsymbol{f}\left(\boldsymbol{E}_{1}\right)}{\left(\boldsymbol{E}_{1}-\boldsymbol{E}_{0}\right)\left(\boldsymbol{E}_{1}-\boldsymbol{E}_{2}\right)\left(\boldsymbol{E}_{1}-\boldsymbol{E}_{2}\right)}+\frac{\boldsymbol{f}\left(\boldsymbol{E}_{2}\right)}{\left(\boldsymbol{E}_{2}-\boldsymbol{E}_{0}\right)\left(\boldsymbol{E}_{2}-\boldsymbol{E}_{1}\right)\left(\boldsymbol{E}_{2}-\boldsymbol{E}_{3}\right)} \\
& +\frac{\boldsymbol{f}\left(\boldsymbol{E}_{3}\right)}{\left(\boldsymbol{E}_{3}-\boldsymbol{E}_{0}\right)\left(\boldsymbol{E}_{3}-\boldsymbol{E}_{1}\right)\left(\boldsymbol{E}_{3}-\boldsymbol{E}_{2}\right)}
\end{aligned}
$$

And in general

$\boldsymbol{f}\left(\boldsymbol{E}_{0}, \boldsymbol{E}_{1}, \ldots, \boldsymbol{E}_{n}\right)=\frac{\boldsymbol{f}\left(\boldsymbol{E}_{0}\right)}{\left(\boldsymbol{E}_{0}-\boldsymbol{E}_{1}\right)\left(\boldsymbol{E}_{0}-\boldsymbol{E}_{2}\right) \ldots\left(\boldsymbol{E}_{0}-\boldsymbol{E}_{n}\right)}+\frac{\boldsymbol{f}\left(\boldsymbol{E}_{1}\right)}{\left(\boldsymbol{E}_{1}-\boldsymbol{E}_{0}\right)\left(\boldsymbol{E}_{1}-\boldsymbol{E}_{2}\right) \ldots\left(\boldsymbol{E}_{1}-\boldsymbol{E}_{n}\right)}+\ldots+\frac{\boldsymbol{f}\left(\boldsymbol{E}_{n}\right)}{\left(\boldsymbol{E}_{n}-\boldsymbol{E}_{0}\right)\left(\boldsymbol{E}_{n}-\boldsymbol{E}_{1}\right) \ldots\left(\boldsymbol{E}_{n}-\boldsymbol{E}_{n-1}\right)}$ 


\section{Conclusion}

Conclusively it can be stated that mathematical functions of the rate of enzymatic reaction are proved the divide differences are symmetrical in all their arguments with temperature, concentration of substrate and concentration of enzyme. Mathematical functions are worked in $\boldsymbol{n}$ limit which is optimum limit.

\section{References}

[1] Nizam Uddin, Enzyme Concentration, Substrate Concentration and Temperature based Formulas for obtaining intermediate values of the rate of enzymatic reaction using Lagrangian polynomial. International Journal of Basic And Applied Sciences, 1(3), 299-302, 2012.

[2] Nizam Uddin, "Estimation of Errors: Mathematical Expressions of Temperature, Substrate Concentration and Enzyme Concentration based Formulas for obtaining intermediate values of the Rate of Enzymatic Reaction," International Journal of Innovation and Applied Studies, vol. 2, no. 2, pp. 153-172, Feb. 2013.

[3] Nizam Uddin, "Interpolate the Rate of Enzymatic Reaction: Temperature, Substrate Concentration and Enzyme Concentration based Formulas using Newton's Method" , "International Journal of Research in Biochemistry and Biophysics", 2( 2), 5-9, 2012.

[4] Cornish-Bowden, Athel, and Athel Cornish-Bowden. Fundamentals of enzyme kinetics. Vol. 3. London: Portland Press, 1995.

[5] Wolfenden, Richard, et al. "The temperature dependence of enzyme rate enhancements." JOURNAL-AMERICAN CHEMICAL SOCIETY 121 (1999): 7419-7420.

[6] Goyal, Manish. Computer Based Numerical \& Statistical Techniques. Firewall Media, 2005 